\title{
Effect of Self-treatment During Rehabilitation Maintenance Phase on Affected Limb Lymphedema in Patients After Breast Cancer Surgery
}

\author{
Litao Zhang, Yanni Li, Yuhua Ma, Rongzhao Lv* \\ Department of Breast Surgery, The First Affiliated Hospital of Jinan University, Guangzhou, China \\ Email address: \\ 190756225@qq.com (Rongzhao Lv) \\ ${ }^{*}$ Corresponding author \\ To cite this article: \\ Litao Zhang, Yanni Li, Yuhua Ma, Rongzhao Lv. Effect of Self-treatment During Rehabilitation Maintenance Phase on Affected Limb \\ Lymphedema in Patients After Breast Cancer Surgery. International Journal of Biomedical Science and Engineering. \\ Vol. 8, No. 2, 2020, pp. 6-10. doi: 10.11648/j.jibse.20200802.11
}

Received: May 16, 2020; Accepted: June 4, 2020; Published: June 20, 2020

\begin{abstract}
Objective: This article aims to discuss the effect of self-treatment during rehabilitation maintenance phase on affected limb lymphedema after surgery for breast cancer. Methods: Therapists for lymphedema gave one course of complex decongestion therapy (CDT) to affected limb lymphedema of 42 patients after operation for breast cancer. After CDT, patients took self-treatment during rehabilitation maintenance phase. At the $1^{\text {st }}, 3^{\text {rd }}, 6^{\text {th }}$, and $12^{\text {th }}$ month after self-treatment, follow-up was conducted for the patients to measure the circumference of the limb, and condition of some lymphedema-related symptoms (such as limitation of shoulder movement, sense of tension, heaviness, needling and numbness). Results: After self-treatment, the circumference of the limb reduced and the lymphedema-induced symptoms improved significantly at the $1^{\text {st }}, 3^{\text {rd }}, 6^{\text {th }}$, and $12^{\text {th }}$ month $(\mathrm{P}<0.05)$. Besides, 12 months after self-treatment, the lymphedema was maintained at stable phase and lymphedema-related symptoms were improved. The incidence of limitation of shoulder movement, sense of numbness, needling, tension, and heaviness reduced significantly compared with that before self-treatment $(\mathrm{P}<0.05)$. Conclusions: Adherence to self-treatment for lymphedema-related symptoms during rehabilitation maintenance phase can reduce the recurrence rate of lymphedema and thus is an effective method for consolidation treatment.
\end{abstract}

Keywords: Breast Cancer, Lymphedema, Complex Decongestive Therapy, Self-treatment, Follow-up

\section{Introduction}

Breast cancer is the most common malignant tumor in females [1]. With the continuous development of medical precision treatment technology in recent years, the long-term survival rate of breast cancer patients has increased significantly, with a 5 -year overall survival rate of more than $85 \%$ [2]. Currently, surgical treatment is a major method for breast cancer. Breast cancer-related lymphedema (BCRL) is one of the most common postoperative complications, with upper limb swelling, heaviness, numbness, shoulder movement limitation, sense of tension and other symptoms which affect the patient's quality of life [3]. Manual lymphatic drainage as a complex decongestion therapy (CDT) is the most effective and conservative treatment method for lymphedema [4]. However, the effect of manual lymphatic drainage will be difficult to maintain and some symptoms may be likely to relapse, if patients do not adhere to self-treatment after discharge. Self-lymphatic drainage with bandaging and wearing a pressure arm sleeve during the maintenance phase is an important measure for follow-up treatment and can consolidate the effect of previous treatment, and it can even be a measure taken by the patient for the rest of life. Our department followed up the effect of self-treatment of 42 patients with upper limb lymphedema after breast cancer surgery during rehabilitation maintenance phase after CDT treatment. The report is as follows.

\section{Data and Methods}

\subsection{General Data}

We selected 42 patients who had affected limb lymphedema after breast cancer operation and received CDT treatment in 
our department from January 2017 to December 2018, including 32 mild and moderate cases and 10 severe cases, aging from 36 to 65 years old, with an average age of $48.03 \pm 2.58$ years old and average BMI of $25.12 \pm 1.24 \mathrm{~kg} / \mathrm{m}^{2}$. Among them, 34 patients underwent modified radical mastectomy for breast cancer, 5 breast conserving surgery plus lymph node dissection and 3 breast conserving surgery plus lymph node biopsy. After operation, 31 cases of lymphedema occurred within 1 year, 6 cases in 1 to 5 years, 3 cases in 5 to 10 years, and 2 cases over 10 years. The Inclusion criteria were: (1) Affected limb lymphedema after breast cancer surgery; (2) Being willing to participate in this study and informed consent, accepting and understanding the content of health education; (3) Residing in Guangzhou; (4) Having received CDT treatment; (5) No metastasis in the neck, upper and lower clavicle, bilateral axillae and bilateral inguinal lymph nodes. Exclusion criteria were: (1) Advanced breast cancer, recurrence, distant metastasis or breast metastatic cancer; (2) Being complicated with major diseases of other organs or severe systemic infection, severe anemia, etc.; (3) Having abnormal cognitive behavior, or using anti-anxiety, anti-depression drugs recently; (4) Having edema caused by thrombus or tumor thrombus; (5) Being unwilling to cooperate with follow-up.

A total of 52 cases of affected limb lymphedema after breast cancer surgery underwent CDT treatment, but there were 2 cases of distant metastasis during follow-up, 3 cases of changing residence, 5 cases of treatment interruption due to economic reasons, so there were 42 patients completing follow-up in the end.

\subsection{Methods}

\subsubsection{Building a Team for Treating Lymphedema}

There was one associate chief physician, one associate chief nurse, two supervisor nurses, two nurses, two therapists for lymphedema in the team, and the department director and head nurse were responsible for the implementation of system and control of quality.

\subsubsection{Establishing a Rehabilitation Communication Group and WeChat Public Account for Patients with Lymphedema}

On the first day, the therapist invited the patients or their family members to join in the WeChat group. At the end of the treatment course, videos about functional exercise, manual lymphatic drainage technology and bandaging operation were shared in the WeChat group. If there were any problems during self-treatment after discharge, patients or their family members could communication with the therapist. The therapists for lymphedema shared educational materials published on breast cancer-related lymphedema websites in the group every week, providing the latest treatment information and knowledge on lymphedema so as to improve the patients' knowledge of lymphedema, providing a communication platform to pose and answer questions. During self-treatment, the condition of upper limb edema was reported to the therapists for lymphedema so as to monitor the self-treatment effect at the early time.

\subsubsection{Key Points of Home Self-treatment}

CDT treatment includes manual lymphatic drainage (MLD), bandaging, functional exercise and skin care, as referred to the method of Foeldi, et al. [5]. (1) MLD operation: the MLD operation should be done as gentle as possible and the order was from the trunk to the limbs, from the healthy side to the affected side, and the regional lymph nodes should be pressed first (supraclavicular, axillary, inguinal lymph nodes); (2) Bandaging: during the treatment, patients were asked to remove and roll up the bandage by themselves, put the bandage materials on the table in the order of use, tear off a small piece of tape, and fix the bandage with the tape immediately after each bandage was wrapped to prevent the bandage from falling off. As the patients were familiar with the procedure, they could finish this operation by themselves at home; (3) Home skin care: itching, erythema, keratosis, etc. were common adverse symptoms during CDT treatment for lymphedema. Belated treatment would reduce the treatment effect or even cause inflammation or concurrent infection, which would aggravate the condition [6]. The patients were informed that if they had skin rashes, itch and other symptoms, they should stop bandaging or wearing pressure sleeves, and only after the skin improved, could they bandage or wear pressure sleeves; (4) Home functional exercise: functional exercise was an important part of the CTD for lymphedema. It could promote local blood circulation and lymphatic return, helping to reduce the edema of the affected upper limb [7]. Functional exercise could be performed during daily life and between work, twice a day and about 20 to 30 minutes each time, but it must be performed under the condition of using a pressure bandage or wearing pressure arm sleeves to achieve the effect, otherwise the edema might deteriorate; (5) Wearing pressure arm sleeve: wearing a pressure arm sleeve was an important measure for subsequent treatment and consolidating the effect of treatment, and it was even a measure that needed to be adopted for the rest of patients' life. Patients needed to wear a suitable pressure arm sleeve, if they did not wear a bandage. Wearing an elastic arm sleeve was simple and convenient, but it could not be taken for a long time; (6) The patients and their family members were instructed to measure the circumference of the limb time after time to ensure the consistency of the measurement; (7) From the first day of treatment, the therapist informed the patients or their family members of manual lymphatic drainage, its operation procedures and precautions, etc. On the first day, the operation procedures were demonstrated and the operation skills were explained. On the second day, therapists taught the patients how to perform lymphatic drainage and bandage dressing till the patients mastered these two operations and patients' family members also received instructions.

\subsubsection{Self-management and Prevention of Lymphedema}

The 18 guidelines for preventing lymphedema proposed by the National Lymphedema Network (HLN) [8] included avoiding venous blood collection, infusion, and blood pressure measurement, wearing protective gloves when 
doing daily housework, keeping the upper limbs hygienic, avoiding injuries to the affected limbs and other suggestions. The therapists for lymphedema introduced the precautions of preventing lymphedema and self-management of limbs at home to the patients and their families.

\subsubsection{Maintenance of Bandage Materials and Elastic Arm Sleeves}

The service life of both the bandage materials and the elastic arm sleeves was limited. It was recommended to wash them with neutral soap every week, and place them in a cool place to avoid exposure to the sun. The patient should wear a bandage and elastic arm sleeve when returning to the clinic so that the therapist could check the tightness of the materials and assess whether they needed to be replaced to ensure their elasticity.

\subsection{Outcome Measurements}

The changes in the circumference of the affected limb and subjective sensation at the $1^{\text {st }}, 3^{\text {rd }}, 6^{\text {th }}$, and $12^{\text {th }}$ month after self-treatment were recorded, and the condition of patients' self-CDT treatment was observed. Telephone or WeChat follow-up was conducted once a week at the first month after discharge and once a month at the second month. If patients felt that the symptoms related to lymphedema worsened and the circumference of affected limb increased during self-treatment, which meant that the treatment effect was bad, they should contact the therapists to increase the number of follow-ups. During subsequent visit, therapists for lymphedema used self-made questionnaires to evaluate symptoms related to lymphedema, including limb swelling, heaviness, tension, numbness, shoulder movement limitation and other edema-related symptoms, and measured the circumference of different parts of the affected limb.

\subsubsection{Measurement of Circumference}

The circumference of the limb at 5 locations (part of the hand between the thumb and the index finger, $5 \mathrm{~cm}$ above the transverse wrist crease, $10 \mathrm{~cm}$ below the cubital crease, $10 \mathrm{~cm}$ above the cubital crease, and $20 \mathrm{~cm}$ above the cubital crease) was measured and recorded.

\subsubsection{Evaluation of Lymphedema-related Symptoms}

The Breast Cancer \& Lymphedema Symptom Experience Index (BCLE-SEI) developed by Fu, et al. in 2012 was used to evaluate the symptoms related to lymphedema, such as swelling of the affected limbs, sense of heaviness, tension, numbness and needling as well as limited shoulder movement, etc. $[9,10]$.

\subsection{Statistical Methods}

We used SPSS13.0 for statistical analysis. The statistical data was expressed by $\bar{\chi} \pm_{\mathrm{S}}$. The $\mathrm{T}$ test was adopted for comparative analysis of the measurement data before and after treatment, and $\chi^{2}$ test for enumeration data, and $\mathrm{P}<0.05$ indicated significant difference.

\section{Results}

\subsection{Comparison of the Effect of Self-treatment on Decongestion in 42 Cases of Affected Limb Lymphedema During Maintenance Phase After CDT}

After CDT, the limb circumference (part of the hand between the thumb and the index finger, $5 \mathrm{~cm}$ above the transverse wrist crease, $10 \mathrm{~cm}$ below the cubital crease, $10 \mathrm{~cm}$ above the cubital crease, $20 \mathrm{~cm}$ above the cubital crease) of 42 patients with affected limb lymphedema was compared before and after self-treatment during the maintenance phase. At the $1^{\text {st }}, 3^{\text {rd }}, 6^{\text {th }}$ month after self-treatment, the circumference decreased and the related symptoms caused by edema improved with a significant difference $(\mathrm{P}<0.05)$. At the $12^{\text {th }}$ month after treatment, the condition of circumference and edema was maintained (see Table 1).

Table 1. Comparison of the Effect of Self-treatment on Decongestion in 42 Cases of Affected Limb Lymphedema during Maintenance Phase after CDT ( $n=42$ ).

\begin{tabular}{lllllll}
\hline Time & $\mathbf{N}$ & $\begin{array}{l}\text { part of the hand } \\
\text { between the thumb and } \\
\text { the index finger }\end{array}$ & $\begin{array}{l}\mathbf{5} \text { cm above the } \\
\text { transverse wrist } \\
\text { crease }\end{array}$ & $\begin{array}{l}\mathbf{1 0} \text { cm below the } \\
\text { cubital crease }\end{array}$ & $\begin{array}{l}\mathbf{1 0} \text { cm above the } \\
\text { cubital crease }\end{array}$ & $\begin{array}{l}\mathbf{2 0} \text { cm above the } \\
\text { cubital crease }\end{array}$ \\
\hline Before self-treatment & 42 & $19.61 \pm 1.13$ & $19.61 \pm 1.90$ & $26.39 \pm 2.39$ & $31.93 \pm 3.36$ & $34.88 \pm 3.78$ \\
$1^{\text {st }}$ month after treatment & 42 & $18.92 \pm 1.08$ & $18.91 \pm 1.82$ & $25.48 \pm 2.13$ & $30.94 \pm 3.23$ & $33.99 \pm 3.21$ \\
$3^{\text {rd }}$ month after treatment & 42 & $18.36 \pm 1.03$ & $18.19 \pm 1.80$ & $24.67 \pm 2.12$ & $29.93 \pm 3.00$ & $33.19 \pm 3.15$ \\
$6^{\text {th }}$ month after treatment & 42 & $17.93 \pm 1.02$ & $17.38 \pm 1.57$ & $23.71 \pm 2.00$ & $28.96 \pm 2.89$ & $32.14 \pm 3.16$ \\
$1^{\text {th }}$ month after treatment & 42 & $17.87 \pm 1.04$ & $17.29 \pm 1.51$ & $23.66 \pm 1.99$ & $28.82 \pm 2.85$ & $32.07 \pm 3.16$ \\
$t_{1}$ & & 13.800 & 15.943 & 13.601 & 16.053 & 8.655 \\
$P_{1}$ & & 0.000 & 0.000 & 0.000 & 0.000 & 0.000 \\
$t_{2}$ & & 15.524 & 16.682 & 15.461 & 15.724 & 15.147 \\
$P_{2}$ & & 0.000 & 0.000 & 0.000 & 0.000 & 0.000 \\
$t_{3}$ & 14.691 & 16.298 & 17.878 & 16.968 & 17.711 \\
$P_{3}$ & 0.000 & 0.000 & 0.000 & 0.000 \\
\hline
\end{tabular}

Note: $\mathrm{t}_{1}, P_{1}$ : before self-treatment vs. $1^{\text {st }}$ month after treatment; $\mathrm{t}_{2}, P_{2}$ : before self-treatment vs. $3^{\text {rd }}$ month after treatment; $\mathrm{t}_{3}, P_{3}$ : before self-treatment vs. $6^{\text {th }}$ month after treatment. 


\subsection{The Incidence of Lymphedema-related Symptoms After Self-treatment During Rehabilitation Maintenance Phase in 42 Patients with Affected Limb Lymphedema}

The incidence of lymphedema-related symptoms such as shoulder movement limitation, numbness, sense of needling, tension, and heaviness in 42 patients with affected limb lymphedema after 12 months of self-treatment during the rehabilitation maintenance phase was significantly lower than that before self-treatment as shown in Table 2. The difference is statistically significant $(\mathrm{P}<0.05)$.

Table 2. Comparison of Incidence of Lymphedema-related Symptoms before and after CDT Self-treatment [n=42, case (\%)].

\begin{tabular}{|c|c|c|c|c|c|}
\hline Related symptoms & $\mathbf{n}$ & Before self-treatment & After self-treatment & $\chi^{2}$ & $P$ \\
\hline Shoulder movement limitation & 42 & $10(23.81)$ & $2(4.76)$ & 44.471 & 0.000 \\
\hline numbness & 42 & $13(30.95)$ & $3(7.14)$ & 34.125 & 0.000 \\
\hline Sense of needling & 42 & $15(35.71)$ & $1(2.38)$ & 36.214 & 0.000 \\
\hline Sense of tension & 42 & $28(66.67)$ & $0(0.00)$ & 26.031 & 0.000 \\
\hline Sense of heaviness & 42 & $19(47.62)$ & $0(0.00)$ & 31.672 & 0.000 \\
\hline
\end{tabular}

\section{Discussion}

BCRL is one of the most common postoperative complications of breast cancer patients, with upper limb swelling, sense of heaviness, numbness, tension, and shoulder movement limitation, which seriously affect the quality of life of patients. CDT is currently the most accurate, effective and safest method for treating lymphedema, which can reduce the arm circumference and the symptoms of edema, improving the quality of life of patients [11]. Previous studies focus on the effects of recent effect of CDT treatment $[12,13]$, and the long-term effect of self-treatment during maintenance period is rarely discussed. In this study, after 1 course of CDT treatment for patients with affected limb lymphedema after breast cancer surgery, the therapists for lymphedema conducted intensive follow-up on the patients for 12 months and found that the circumference of the affected limb of the patients reduced after 6 months of self-treatment, with a significant difference $(\mathrm{P}<0.05)$. After 12 months of self-treatment, the circumference of the affected limb reduced slightly, which meant that the edema remained in a stable period. There was no case of recurrence of edema, and improvement on patient's subjective feelings including limited shoulder movement, numbness, and sense of needling, tension and heaviness. Chen Jiajia et al. [14] found that, in 102 patients with lymphedema who had been treated by CDT, self-treatment helped to improve the condition of the circumference of the affected limb and the patients' subjective sensation, and there was no cases of recurrence, which is consistent with the results of this study.

Studies have found $[15,16]$ that to strengthen health education for patients and their families in identifying early symptoms of lymphedema, recovery, treatment and prognosis, and to strengthen postoperative follow-up and supervision help patients find symptoms related to lymphedema as early as possible and promote them to take active preventive measures, improve self-management capabilities and improve compliance with timely medical treatment. During self-treatment in the rehabilitation maintenance period, our lymphedema treatment team regularly shared information on lymphedema in the WeChat group to encourage self-treatment and facilitate follow-up. At each follow-up visit, by measuring the circumference of the limb and evaluating the condition of patient's lymphedema-related symptoms, we informed patients of the dangerous behavior that would cause lymphedema, and taught patients to recognize the symptoms and signs related to lymphedema. As a result, we help them improve their attention, self-identification ability, and compliance with self-treatment.

Studies have also found that $[17,18]$ pressure arm sleeves had a certain effect on relieving symptoms of lymphedema, but the long-term effect was not obvious. Melam et al. [19] found that adherence to using elastic bandages could reduce the circumference of the affected limb and consolidate previous treatment effect. Another report pointed out that [20] patients' lack of professional supervision and assistance during the follow-up and self-treatment would cause decrease in patient's compliance to treatment, resulting in recurrence of lymphedema. On the first day of intensive treatment, our therapists demonstrated the operation procedures and techniques and explained the main points of the operation. On the second day, we began to instruct patients and their families on the operation. The pressure treatment in the one year of self-treatment during rehabilitation maintenance period mainly includes bandaging, assisted with pressure arm sleeves. At the same time, during the follow-up, the therapists for lymphedema establishes an interactive platform with the patients to strengthen supervision, answer the patient's questions at any time, provide professional information, and encourage the patient to insist on self-treatment. Besides, the platform helps increase the patient's self-management ability and ensure the continuity of treatment to maintain the best therapeutic effect, improve the subjective feeling of patients and reduce the recurrence rate of lymphedema.

\section{Conclusions}

In conclusion, lymphedema is one of the most common complications after breast cancer surgery. Once lymphedema occurs, it is often a lifelong condition and requires long-term treatment and care. The effect of CDT is clear. It can reduce the arm circumference and the symptoms of edema, and improve patients' subjective feelings and their quality of life. Although lymphedema cannot be cured, adherence to self-treatment during the rehabilitation maintenance period 
can prevent the recurrence of edema. As the sample size of this study is small, and the follow-up lasts only 1 year, we will increase the sample size and follow-up time in the future to conduct a study on the long-term effect.

\section{References}

[1] Bray F, Ferlay J, Soerjomataram I, et al. "Global cancer statistics 2018: GLOBOCAN estimates of incidence and mortality worldwide for 36 cancers in 185 countries" [J]. $C A$ Cancer J Clin, 2018, 6 (68): 394-424.

[2] Zhang Shunkang, Sun Liyun, Chen Gang. "Correlation analysis of upper limb lymphedema and the outcome of axillary lymph node surgery in breast cancer patients treated with postmastectomy radiation therapy" [J]. China Oncology, 2018, 28 (01): 55-61.

[3] Liu Fenghua. "Breast Cancer Related Lymphedema: Pathogenesis, Incidence Rates, Diagnostic Techniques, Treatment Paradigms and Risk Reduction" [D]. Suzhou: Soochow University, 2014.

[4] International Society of Lymphology. "The diagnosis and treatment of peripheral lymphedema: 2009 consensus document of the international society of lymphology" $[\mathrm{J}]$. Lymphology, 2009, 42 (2): 51-60.

[5] Lam VB, Czyz CN, Wulc AE. "The brow-eyelid continuum: an anatomic perspective" [J]. Clin Plast Surg, 2013, 40 (1): $1-19$.

[6] Felmerer G, Sattler T, Lohrmann C, et al. "Treatment of various secondary lymphedemas by microsurgical lymph vessel transplantation" [J]. Microsurgery, 2012, 32 (3): 171-177.

[7] $\mathrm{He}$ Yingjun. "Research Progress on Meridian Massage Combined with Progressive Rehabilitation Exercise in Improving Limb Function of Patients after Breast Cancer Surgery" [J]. Chinese Nursing Research, 2015, 29 (19): 2311-2313.

[8] Kang Y, Jang D H, Jeon JY, et al. "Pressure monitoring of multilayer in elastic bandaging and the effect of padding in breast cancer -related lymphedema patients" [J]. Am J Phys Med Rehabil, 2012, 91 (9): 768-773.

[9] $\mathrm{Fu} \mathrm{MR}$, Ridner SH, Armer J. "Post-breast cancer. Lymphedema: part” [J]. Am J Nurs, 2009, 109 (7): 48-54.
[10] Li Chunyue. "The effectiveness of a rehabilitation exercise program for postoperative patients with breast cancer related lymphedema" [D]. Hebei University, 2016.

[11] Luan Jie, Mu Dali, et al. "Recent advances on the treatment of breast cancer-related lymphedema" [J]. Chinese Journal of Plastic Surgery, 2018, 34 (7): 578-582.

[12] Chen Jiajia, Wang Li, Yu Ziyou, et al. "Manual Lymph Drainage for the Treatment of Upper Limb Lymphedema after Breast Cancer Treatment" [J]. Journal of Tissue Engineering and Reconstructive Surgery, 2015, 11 (05): 310-312.

[13] Yin Ping. "Observation on the Effect of Applying Limb Compression and Manual Lymphatic Drainage Massage on Patients with Affected Limb Edema after Breast Cancer Operation" [J]. Journal of Nursing, 2012, 19 (14): 53-54.

[14] Chen Jiajia, Wang Li, Han Linghua, et al. "Effects of Complex Decongestive Therapy Combined with Manual Lymphatic Drainage in the Treatment of Lymphedema of Limbs" [J]. Journal of Tissue Engineering and Reconstructive Surgery, 2017, 13 (06): 322-324+327.

[15] Shen Yi, Qiang Wanmin, Tang Lei, et al. "A Qualitative Study on the Reasons for Delayed Treatment in Patients with Severe Upper Limb Lymphedema after Breast Cancer Surgery" [J]. Journal of Nurses Training, 2016, 31 (18): 1699-1702.

[16] Fu MR, Chen CM, Haber J, et al. "The effect of providing information about lymphedema on the cognitive and symptom outcomes of breast cancer survivors" [J]. Ann Surg Oncol, 2010, 17 (7): 1847-1853

[17] Jiang Liuya, Xie Na, Ruan Xiangmei, et al. "Effects of pressure cuff assisted lymphatic drainage on upper limb lymphedema in patients after breast cancer surgery" [J]. Chinese Journal of Rehabilitation Medicine, 2018, 33 (04): 409-412.

[18] Shao Y, Zhong DS. "Manual lymphatic drainage for breast cancer-related lymphedema" [J]. Eur J Cancer Care (Engl), 2017, 26 (5): 1-5.

[19] Melam GR, Burgadda S, Slhusaini AA, et al. "Effect of complete decongestive therapy and home program on health-related quality of life in post mastectomy lymphedema patients" [J]. BMC Womens Health, 2016.16: 23.

[20] Qiu Jiajia, Li Ping. "Effect of Compression Elastic Bandage on Improving Upper Limb Lymphedema in Postoperative Breast Cancer Patients" [J]. Shanghai Nursing, 2019, 19 (09): 34-38. 